\title{
Teletherapy for subfoveal choroidal neovascularisation of age-related macular degeneration: results of follow up in a non-randomised study
}

\author{
P M Hart, U Chakravarthy, G MacKenzie, D B Archer, R F Houston
}

\begin{abstract}
Aim-A preliminary report indicated stable or improved vision in 12 of 19 patients with subfoveal choroidal neovascularisation treated with 12 or 15 Gy of $6 \mathrm{MV}$ photons to the affected macula after an average follow up of 18 months. Here the prolonged follow up findings in this group of treated patients is reported which was further increased to 41 .

Methods-Forty one patients with subfoveal choroidal neovascularisation were treated with 10,12 , or 15 Gy of $6 \mathrm{MV}$ photons to the macula of the affected eye. Thirteen eyes of 12 patients were also observed as a non-randomised comparison group.
\end{abstract}

Results-At 12, 18, and 24 months of follow up the mean change in visual acuity in eyes treated with radiotherapy was less than 1 Bailey-Lovie line from that measured at presentation. By contrast, the eyes in the comparison group lost 3.7 lines of acuity at 12 months which increased to 4.5 at 24 months. These differences were highly significant at each of the time points. When initial visual acuity was taken into account, treated eyes lost on average $12 \%$ of baseline acuity throughout follow up, whereas eyes belonging to the untreated group lost $50 \%$ of baseline acuity at 1 year, and $75 \%$ at 2 years. There was no significant difference in visual outcome between the three dose regimens used, which may simply be a reflection of the small sample size in each group. There was no evidence of radiation induced retinopathy or optic neuropathy in any treated patients.

Conclusions-Teletherapy appeared to have a treatment effect in eyes with subfoveal choroidal neovascularisation resulting in maintained visual function without significant radiation induced morbidity.

(Br F Ophthalmol 1996;80:1046-1050)

The exudative form of age-related macular degeneration (ARMD) is associated with a particularly poor visual prognosis and accounts for $88 \%$ of ARMD sufferers who are registered blind. ${ }^{1}$ Laser photocoagulation remains the mainstay of treatment for those choroidal neovascular membranes (CNVM) which are lo- cated outside the foveal avascular zone. ${ }^{23}$ In practice, however, the majority of CNVM at initial presentation have some degree of foveal involvement. ${ }^{4}$ Although in the long term, laser photocoagulation of subfoveal membranes results in better preserved central visual function in treated cases when compared with no treatment, it is often marginal and there is a significant and immediate post-treatment fall in acuity. ${ }^{5}$ As the visual benefit does not become evident until 18 months later, this treatment option is unacceptable to most ARMD sufferers. In addition, even when patients with foveal involvement are deemed suitable for laser photocoagulation, a recent study has shown that only $26 \%$ of all patients with exudative ARMD meet the Macular Photocoagulation Study Group (MPS) criteria. ${ }^{6}$ Consequently, a significant number of patients with CNVM are managed conservatively - that is, the disease process is allowed to take its natural course.

We have shown previously in a pilot study consisting of 19 patients with neovascular ARMD that focal radiotherapy resulted in stabilisation and maintenance of central visual function during an average follow up time of 1 year. ${ }^{8}$ In this paper we report on the long term visual outcome post-radiotherapy of patients with subfoveal CNVM.

\section{Subjects and methods}

Patients deemed unsuitable for laser photocoagulation under the MPS extrafoveal CNV criteria were referred to a special interest clinic in a general ophthalmic outpatient department. All patients were over the age of 60 and had CNVM due to ARMD. Fifty three patients with subfoveal neovascularisation on fluorescein angiography were identified for inclusion into the study. The angiograms of all these patients showed early leakage of dye seen as hyperfluorescence which increased in intensity and area and which involved the foveal avascular zone. The patients were fully informed of the nature of their condition and treatment options available. From January 1992 those patients who fitted the MPS criteria for foveal ablation $^{6}$ were offered this treatment but all declined. Any patient with pre-existing ocular disease (for example, glaucoma, high myopia, chronic inflammatory, or neoplastic disorders) was excluded as were those with systemic disorders (diabetes, uncontrolled hyperten- 
Table 1 Comparison of baseline characteristics of patients

\begin{tabular}{llll}
\hline & $\begin{array}{l}\text { Treatment } \\
\text { group }(n=41)\end{array}$ & $\begin{array}{l}\text { Control } \\
\text { group } \\
(n=13)\end{array}$ & $p$ Value \\
\hline Age (years): & & & \\
$\quad$ Mean (SE) & $73.34(1.10)$ & $71.7(1.67)$ & 0.45 \\
Median & 73 & 73 & $0.46^{\star}$ \\
Min & 60 & 63 & \\
$\quad$ Max & 88 & 81 & \\
Visual acuity & & & \\
(logMAR): & & $0.81(0.08)$ & 0.23 \\
Mean (SE) & $0.95(0.06)$ & 0.78 & $0.27^{\star}$ \\
$\quad$ Median & 1.00 & 0.48 & \\
Min & 0.30 & 1.30 & \\
Max & 1.78 &
\end{tabular}

^Indicates non-parametric comparison.

sion) or a known life threatening disease at enrolment into the study. Informed consent to participate in the radiotherapy study was obtained in all treated cases. Those patients who declined radiotherapy were followed up as a non-randomised comparison group (a total of 41 eyes received radiotherapy and 13 eyes of 12 patients were followed up as controls).

All recruited patients were subjected to a detailed ophthalmic examination including slit-lamp biomicroscopy. Fully corrected distance visual acuity was measured on the Snellen chart with the patient at 6 metres. If the patient was unable to read the $6 / 60$ $(20 / 200)$ line, he or she was moved forward in 1 metre steps until they were able to read the top two lines (for example, 2/36). The log of the minimum angle of resolution was calculated and used for all statistical analysis.

Before embarking on the study, approval from the ethics committee of the host institution was obtained. An immediate pretreatment or pertreatment fluorescein angiogram (that is, before or within 24 hours of commencing radiotherapy) was obtained in all but the initial five patients. If a delay of 2 weeks or more occurred between ophthalmic examination and the inception of radiotherapy a further visual acuity measurement was made. Radiotherapy was carried out as previously described. ${ }^{8}$ In brief, the patient was fitted with a custom made beam direction shell and a computed tomography scan was performed. Treatment plans were generated, prescribed to the $90 \%$ isodose which encompassed the macula of the affected eye. Three dose regimens were used, $10 \mathrm{~Gy}$ in five divided doses over 5 days, $12 \mathrm{~Gy}$ in six divided doses over 8 days, and $15 \mathrm{~Gy}$ in five divided doses over 5 days. The nominal standard dose (NSD) (rets) ${ }^{9}$ was calculated using the formula

$$
\mathrm{NSD}=\mathrm{TD} \div \mathrm{N}^{24} \times \mathrm{T}^{11}
$$

(where TD is the total dose, $\mathrm{N}$ is the number of fractions, and $T$ is the duration of days spanned by treatment) and was below 820 rets for each of the schedules used. After radiotherapy the patients were reviewed at $3,6,12$, 18,24 months, and then yearly. Visual function was assessed at every visit and angiography scheduled for the $3,6,12$, and 24 month visits.

The primary outcome variable chosen for analysis was the change in visual acuity between baseline and each follow up examination. Data pertaining to changes in visual acuity refer to Bailey-Lovie lines unless otherwise stated. The longitudinal change from baseline was analysed by univariate statistical methods at each follow up visit and by appropriate multivariate statistical techniques which allowed for the correlation between intraindividual visual acuity measurements over time. Although the follow up information was largely complete, 29 (17.9\%) visual acuity measurements were missing (11 (6.8\%) at 18 month review and 18 $(11.1 \%)$ at 24 month review) at the time of analysis. These values were imputed by exploiting the observed relations among the visual acuity measurements of patients whose data were complete. The imputation was carried out separately in the treatment and control groups by, for example, regressing visual acuity measurements made at time $t_{12}$ on those made at $t_{0}$ and using the resulting regression equation to predict the values missing at time $t_{12}$. Such methodology is well established. ${ }^{10} \mathrm{~A}$ similar strategy was employed between times $t_{12}$ and $t_{24}$.

\section{Results}

AT BASELINE

Age and visual acuity (VA) measured on entry to the study were compared in the treatment (n $=41)$ and untreated $(n=13)$ groups (Table 1). On average, treated patients were 1.5 years older than untreated (73.3 $v 71.7$ years) but this difference did not reach statistical significance. Baseline VA was slightly poorer in treated patients compared with untreated ( median $=1 v$ median $=0.78 \log$ MAR) but as with age this difference was not formally significant. All the angiograms of patients showed classic choroidal neovascularisation

Table 2 Grouped visual acuity over time in treated and untreated patients

\begin{tabular}{|c|c|c|c|c|c|c|c|c|c|c|c|c|}
\hline \multirow[b]{3}{*}{ Time (months) } & \multicolumn{6}{|c|}{ Treatment group } & \multicolumn{6}{|c|}{ Comparison group } \\
\hline & \multicolumn{2}{|c|}{ Good } & \multicolumn{2}{|c|}{ Moderate } & \multicolumn{2}{|c|}{ Poor } & \multicolumn{2}{|c|}{ Good } & \multicolumn{2}{|c|}{ Moderate } & \multicolumn{2}{|c|}{ Poor } \\
\hline & $n$ & $\%$ & $n$ & $\%$ & $n$ & $\%$ & $n$ & $\%$ & $n$ & $\%$ & $n$ & $\%$ \\
\hline $\begin{array}{r}0 \\
12 \\
18 \\
24 \\
36 \\
48\end{array}$ & $\begin{array}{r}11 \\
11 \\
7 \\
6 \\
4 \\
4\end{array}$ & $\begin{array}{l}26.8 \\
26.8 \\
21.9 \\
23.1 \\
16.7 \\
-\end{array}$ & $\begin{array}{r}20 \\
17 \\
14 \\
11 \\
13 \\
3\end{array}$ & $\begin{array}{l}48.8 \\
41.5 \\
43.8 \\
42.3 \\
54.2 \\
-\end{array}$ & $\begin{array}{r}10 \\
13 \\
11 \\
9 \\
7 \\
2\end{array}$ & $\begin{array}{l}24.4 \\
31.7 \\
34.4 \\
34.6 \\
29.2 \\
-\end{array}$ & $\begin{array}{l}6 \\
1 \\
0 \\
0 \\
0 \\
0\end{array}$ & $\begin{array}{r}46.2 \\
7.7 \\
0.0 \\
0.0 \\
- \\
-\end{array}$ & $\begin{array}{l}5 \\
7 \\
6 \\
4 \\
1 \\
2\end{array}$ & $\begin{array}{l}38.5 \\
53.9 \\
54.5 \\
40.0 \\
- \\
-\end{array}$ & $\begin{array}{l}2 \\
5 \\
5 \\
6 \\
3 \\
2\end{array}$ & $\begin{array}{l}15.4 \\
38.5 \\
38.5 \\
60.0 \\
- \\
-\end{array}$ \\
\hline 48 & \multicolumn{2}{|c|}{ Snellen } & \multicolumn{2}{|c|}{ Bailey-Lovie } & & & & & & & & \\
\hline $\begin{array}{l}\text { Good acuity } \\
\text { Moderate acuity } \\
\text { Poor acuity }\end{array}$ & \multicolumn{2}{|c|}{$\begin{array}{l}6 / 6 \text { to } 6 / 24 \\
6 / 36 \text { to } 5 / 60 \\
4 / 60 \text { to } 1 / 60\end{array}$} & \multicolumn{2}{|c|}{$\begin{array}{l}0.0 \text { to } 0.78 \\
0.78 \text { to } 1.1 \\
1.2 \text { to } 1.78\end{array}$} & & & & & & & & \\
\hline
\end{tabular}


Table 3 Comparison of the mean change in visual acuity between treatment and control groups at follow up

\begin{tabular}{llll}
\hline & \multicolumn{3}{l}{ Mean difference (SE) from baseline at } \\
\cline { 2 - 4 } Comparison & 12 months & 18 months & 24 months \\
\hline Treatment group & $+0.03(0.07)$ & $+0.02(0.07)$ & $+0.05(0.07)$ \\
Control group & $+0.37(0.12)$ & $+0.44(0.11)$ & $+0.45(0.11)$ \\
Difference (C - T) & $+0.34(0.14)$ & $+0.42(0.14)$ & $+0.40(0.14)$ \\
Significance & $\mathrm{p}<0.03$ & $\mathrm{p}<0.01$ & $\mathrm{p}<0.01$ \\
\hline
\end{tabular}

NB Positive signs indicate worsening visual acuity.

(early well defined leakage occurring within 30 seconds of dye injection). In addition, the angiograms of 16 treated and six untreated patients showed late leakage of indeterminate origin indicative of occult neovascularisation.

\section{AT FOLLOW UP}

Follow up times for treated and untreated patients ranged between 12 and 55 months. Distribution of visual acuity in treated and untreated eyes is shown in Table 2 . Acuity was subdivided into good (6/6 to $6 / 24)$, moderate (6/36 to $5 / 60)$, and poor ( $4 / 60$ or worse) using similar categories to those described by the MPS.

Table 2 shows the distribution of visual acuity seen in treated and untreated patients over the 48 month follow up period. However, follow up beyond 24 months is incomplete. Inevitably the percentages in each category in the control group have large standard errors and no attempt has been made to compare the groups formally using this categorisation. However, the table does show that the majority of treated patients had either good or moderate visual acuities throughout the follow up period in contrast with subjects in the control group.

Table 3 shows the change in visual acuity measured from baseline to each of three follow up examinations at 12,18 , and 24 months respectively when follow up data were most complete. In compiling Table 3 we have computed the arithmetic difference in visual acuity as: follow up minus baseline (for example, $\mathrm{VA}_{12}-\mathrm{VA}_{0}$ ) for each subject. This quantitative approach makes better use of the data than does the categorical analysis given in Table 1 . Over the 2 year period the mean change in VA from baseline in those treated with radiotherapy was almost stationary. At none of the time points studied was there evidence of a significant departure from the null hypothesis value of zero change. However, the pattern observed in the untreated group is strikingly different. A year after entry to the study visual acuity had significantly deteriorated from baseline and continued to decline further although at a reduced rate until 18

Table 4 Comparison of the mean percentage change in visual acuity between treatment and control groups at follow up

\begin{tabular}{llll}
\hline & \multicolumn{4}{l}{ Mean \% difference (SE) from baseline at } \\
\cline { 2 - 4 } Comparison & 12 months & 18 months & 24 months \\
\hline Treatment group & $+12.7(10.2)$ & $+12.5(10.1)$ & $+11.6(9.6)$ \\
Control group & $+52.7(16.3)$ & $+67.4(16.0)$ & $+74.8(17.9)$ \\
Difference (C - T) & $+39.9(20.3)$ & $+54.9(20.1)$ & $+63.2(19.8)$ \\
Significance & $\mathrm{p}=0.054$ & $\mathrm{p}<0.01$ & $\mathrm{p}<0.01$ \\
\hline
\end{tabular}

NB Positive signs indicate worsening visual acuity. months. No further deterioration in acuity was observed between 18 and 24 months in the untreated group. However, on comparing treated and untreated groups at each of the 12 , 18 , and 24 month stages of follow up, the deterioration in visual acuity was always significantly worse in the latter (Table 3 ).

In order to allow for the small but observed differences in baseline visual acuities in treated and untreated groups we have also analysed the percentage change from baseline (Table 4). As visual acuity is shown in the $\log M A R$ scale, which is an arithmetic progression with better acuities scoring low numbers and worse acuities scoring high numbers, any given change will appear greater if analysed as a proportion of the initial visual acuity when the latter is good. Although the control group did have a better mean initial acuity the observed percentage differences at all times shown in Table 4 are markedly greater than those of the treatment group.

As before, the deterioration among those treated is not significantly different from zero at any of the follow up examinations. By comparison, visual acuity in untreated patients had worsened on average by $52.7 \%$ of the baseline value at 12 months and by $74.8 \%$ at 24 months.

All of the statistical tests described above were confirmed by computing their appropriate non-parametric equivalents and the interpretation of the data is unequivocal using either method of analysis. In addition, multivariate regression analyses were also performed (on the data summarised in Tables 3 and 4) and again the findings were unchanged.

\section{APPEARANCE AND PROGRESSION}

The macular appearance and angiographic signs associated with regression of CNVM following radiotherapy have been described in detail previously. Ten treated patients developed fresh neovascularisation at a site remote from the original CNVM. In one patient this occurred 3 years after treatment resulting in a fall in acuity of 3 lines. In the remaining nine patients fresh neovascularisation occurred between 4 and 10 months after treatment (mean 7.1 (SD 2.4) months). Recurrences were similar whether patients were treated with 10,12 , or $15 \mathrm{~Gy}$ although numbers in each of the groups are too small for definitive conclusions to be reached.

\section{POSSIBLE ADVERSE REACTIONS}

Throughout the duration of the study, patients were monitored for any possible adverse side effects which could be attributed to radiotherapy. Transient conjunctival irritation was reported by one patient with resolution within 3 weeks from radiotherapy and thereafter this patient has remained asymptomatic. Another patient suffered transient alopecia areata involving an area $2 \mathrm{~cm}$ in diameter at the beam exit point. Both these patients received $15 \mathrm{~Gy}$. Significant progression of cortical and posterior subcapsular lens opacities with accompa- 
Table 5 Classification of visual acuity and lesion size $(n=41)$

\begin{tabular}{llll}
\hline & \multicolumn{4}{l}{ Lesion size (MPS disc areas) } \\
\cline { 2 - 4 } Visual acuity & Small $(\leq 1)$ & Medium $(>1$ to $\leq 2)$ & Large $(>2)$ \\
\hline Poor $(\leq 6 / 60)$ & $6(1)$ & $6(0)$ & $9(0)$ \\
Moderate $(6 / 36$ to $6 / 48)$ & $4(1)$ & 0 & $6(2)$ \\
Good $(\geq 6 / 24)$ & $7(0)$ & 0 & $3(1)$ \\
\hline
\end{tabular}

nying loss of acuity was observed in the treated eyes of two patients (both had received $15 \mathrm{~Gy}$ ) 36 months after treatment. Cataract extraction and intraocular lens insertion have been carried out in both these patients. After surgery, vision returned to the level measured before the onset of lens opacities. Radiation induced retinal vasculopathy (microvascular abnormalities, leakage, and cotton wool spots) or optic neuropathy (disc pallor) were not observed clinically. Angiograms were scrutinised for evidence of retinal microvascular abnormalities and none was found.

\section{COMPARISON WITH MPS}

A recent report from the MPS subdivided patients into groups on the basis of the initial lesion size and visual acuity in order to establish a relation between these variables and visual outcome after foveal ablation. ${ }^{11}$ A 6 line drop in VA (quadrupling of the visual angle) was identified by the MPS as a serious visual event and used to define visual outcome in treated (foveal ablation) and control (untreated) patients. In an attempt to compare the results of radiotherapy with those of the MPS foveal ablation study we have subdivided our treated patients into similar categories based on the baseline entry criteria of visual acuity and lesion size (Table 5). This shows the number of patients in each subgroup and in parentheses the number who suffered a 6 line drop in acuity from baseline. In total, only five $(12 \%, 95 \%$ CI $=(1.8 \%-22.4 \%))$ patients treated with radiotherapy suffered a 6 line fall in acuity after 24 months of follow up. Overall, $23 \%$ of MPS study patients suffered a 6 line loss of acuity at 24 months. However in the MPS best outcome group $16 \%, 6 \%$, and $19 \%$ of patients suffered a 6 line drop at 12,24 , and 36 months respectively. Thus, radiotherapy treated patients appear to fare no worse than the best outcome group of the MPS.

\section{Discussion}

Various novel treatments have been proposed in recent years for the management of CNVM untreatable by laser. Interferon alfa-2a which is a potent inhibitor of vascular endothelial cell proliferation, and migration in culture, has been used systemically in the treatment of subfoveal CNVM..$^{1213}$ To date this treatment option has not proved significantly effective and it is also associated with severe secondary effects which may be local or systemic. ${ }^{14}$ Other experimental therapies involve the use of thalidomide, retinoids, and amiloride ${ }^{15}$ all of which are known to possess antiangiogenic properties. The results of such treatments are as yet unavailable. Surgical excision of CNVM has been attempted. ${ }^{16}{ }^{17}$ but the outcome is significantly better in younger patients with presumed ocular histoplasmosis rather than ARMD. ${ }^{16}$ Transposition of the retina has also been considered; however, these are all highly invasive procedures and it is doubtful whether central visual function can be preserved or improved by such drastic surgery.

Radiotherapy is attractive as it can be delivered to a precise location, it is non-invasive, and has no systemic side effects at low doses. Since the publication of our original studies on radiotherapy in ARMD there has been increasing interest in this treatment modality. External beam radiation as employed by other centres in the management of CNVM has included dose regimens ranging from $8 \mathrm{~Gy}$ to $24 \mathrm{~Gy} .{ }^{1819}$ The fractionation schedules varied considerably and fraction sizes as large as 8 Gy have been used. ${ }^{18} 19$ Where cyclotron facilities were available proton beam irradiation has been used which, with its highly collimated beam and sharply defined Bragg-Peak effect, has theoretical advantages in the treatment of CNVM. $^{20}$ Alternatively brachytherapy rather than teletherapy has also been tried with some investigators using palladium-103 or strontium-90 plaques ( $\beta$ emitters) designed to deliver doses between 12.5 and $15 \mathrm{~Gy}$ to the region of the CNVM. ${ }^{2122}$ Most of these recent reports suggest a positive treatment effect in the short term. Although no adverse effects have been reported in any of these studies it should be noted that the follow up times have in general been less than 1 year.

In our studies we used doses ranging from $10 \mathrm{~Gy}$ to $15 \mathrm{~Gy}$ in fractions of 2 or $3 \mathrm{~Gy}$. Although our initial patients were treated with 3 Gy fractions we modified our treatment regimen to administer 2 Gy fractions only. This change was instituted as published evidence exists to show that the risk of optic neuropathy, ${ }^{923}$ and radiation induced retinal vasculopathy ${ }^{24}$ is significantly increased with larger fraction sizes. In a seminal piece of work Harris and Levene ${ }^{9}$ showed that there was a significant increase in the risk of visual loss not only with fraction sizes exceeding 2 Gy but also when the NSD exceeded 1500 rets. In practice, the majority of radiotherapists do not prescribe in fraction sizes in excess of 2 Gy particularly when the field of radiotherapy includes the brain and the eye. These are important considerations as ARMD is a non-life threatening condition and since central vision is already poor in ARMD sufferers, it would be questionable clinical practice to compromise peripheral visual function by any therapeutic intervention. In this context it should be noted that at least one investigator has reported sight threatening retinopathy in a patient with dysthyroid ophthalmopathy treated with external beam radiation at a total dose of $20 \mathrm{~Gy}$ given in 10 fractions over periods of 10 to 14 days. ${ }^{25}$ Our calculations based on the information provided in this paper show that the NSD in these patients was in the region of 1120 to 1160 rets and thus we feel it prudent to restrict the dose and fraction size to ensure a ret value below 1000.

The present study which is an extension of work previously described ${ }^{8}$ has shown a 
sustained positive benefit on central visual function in patients with subfoveal CNVM. At 12,18 , and 24 months after radiotherapy the mean change in visual acuity from baseline was less than 1 line indicating stabilisation of visual function. This contrasts significantly with the visual acuity losses experienced by the comparison group in whom a loss of 3.7 lines occurred at 12 months increasing to 4.4 at 18 and 4.5 lines at 24 months. These findings in our untreated patients are almost identical to that reported in the control patients of the subfoveal MPS study. It is noteworthy that the MPS reported that at 24 months $23 \%$ of treated (foveal laser) and $37 \%$ of untreated patients had lost 6 or more lines of acuity. A later reanalysis of their data identified a subgroup of patients with small initial lesion size and moderate or poor visual acuity $(6 / 36$ or better) or medium lesion size and poor initial acuity who appeared to respond favourably to treatment and thus constituted the best outcome group. In the present study despite subdivision of patients treated with radiotherapy into similar categories it was not possible to identify a best outcome group possibly because of small numbers. None the less, a 6 line drop in acuity was only seen in five patients $(12 \%, 95 \%$ CI $=(1.8 \%-22.4 \%))$ from the entire heterogeneous radiotherapy group.

In the light of this same MPS report we subjected our data to further analysis which took into account the baseline acuity measurements. Table 3 indicates the mean percentage change from baseline showing that radiotherapy treated eyes lose approximately $12 \%$ of initial acuity by 1 year. Thereafter, further losses in visual acuity did not occur. However, a $50 \%$ loss of acuity occurred in the untreated group by 12 months increasing to $75 \%$ by 24 months. Our statistical analysis confirmed the lack of significant difference in baseline characteristics in treated and untreated patients (Table 1). Although there is considerable potential for bias to enter non-randomised studies, analysis of the observed difference in visual loss between the two groups suggests that treatment is effective.

The effect of dose $(10,12$, and $15 \mathrm{~Gy})$ on the change in visual acuity from baseline was examined. No significant difference in outcome between any of the three different dose regimens was detected, a finding which was not unexpected given the small numbers of patients involved. However, statistical analysis confirmed that visual outcome in patients receiving any one of these dose regimens was better than that observed in the control group.

This study has shown that radiotherapy significantly alters the natural history of disciform scarring in subfoveal CNVM and that the beneficial effects are maintained over a prolonged follow up. A multicentre randomised controlled study to examine the effect of radiotherapy on subfoveal CNVM is now under way.

The support of the Wellcome Trust and the Medical Research Council is gratefully acknowledged.

1 Kahn HA, Moorehead HB. Statistics on blindness in the model reporting area 1969-1970. United States Department of Health, Education and Welfare Publication No NIH) 73-427. Washington DC: US Government Printing Office, 1973.

2 Macular Photocoagulation Study Group. Argon laser photocoagulation for neovascular maculopathy. Three year
results from randomized clinical trials. Arch Ophthalmol 1986;104:694-701.

3 Bird AC. Recent advances in the treatment of senile disciform macular degeneration by photocoagulation. $\mathrm{Br} f$ disciform macular degeneration

4 Bressler NM, Bressler SB, Gragoudas EG. Clinical characteristics of choroidal neovascular membranes. Arch Ophthalmol 1987;105:209-31

5 Rodger FC. Some practical conclusions following a longitudinal study of common macular lesions. Trans Ophthalmol Soc UK 1982;102:187-97

6 Macular Photocoagulation Study Group. Laser photocoagulation of subfoveal neovascular lesions in age-related macular degeneration. Arch Ophthalmol 1991;109:122031 .

7 Moisseiev, J, Ahalel A, Masuri R, Treister G. The impact of macular photocoagulation study results on the treatment of exudative age-related macular degeneration. Arch Ophthal mol 1995;113:183-9.

8 Chakravarthy U, Houston RF, Archer DB. Treatment of age-related subfoveal neovascular membranes by teletherapy: a pilot study. Br f Ophthalmol 1993;77:265-73.

9 Harris JR, Levene MB. Visual complications following irradiation for pituitary adenomas and craniopharyngiomas. Radiology 1976;120:167-71.

10 Tanner M. Tools for statistical inference. Hayword: IMS, 1991.

11 Macular Photocoagulation Study Group. Visual outcome after laser photocoagulation for sub-foveal choroidal neovascularisation secondary to age-related macular deneovascularisation secondary to age-related macular devisual acuity. Arch Ophthalmol 1994;112:480-8

12 Gillies MC, Sarks JP, Beaumont PE, Hunyor AB, McKay D, Kearns $M$, et al. Treatment of choroidal neovascularisation in age-related macular degeneration with interferon alfa-2a and alfa-2b. Br $\mathcal{F}$ Ophthalmol 1993;77:759-65.

13 Kirkpatrick JNP, Dick AD, Forrester JV. Clinical experience with interferon alfa $2 \mathrm{a}$ for exudative age-related macular degeneration. Br f Ophthalmol 1993;77:766-70.

14 Tang Y. Interferon friend or foe. Arch Ophthalmol 1995;113: 987.

15 Guyer DR. Experimental therapies for exudative age-related macular degeneration. American Academy Abstracts 1995: 60-7.

16 Thomas MA, Grand MG, Williams DF, Lee CM, Pesin SR Lowe MA. Surgical management of subfoveal choroida neovascularization. Ophthalmology 1992;99:952-68.

17 Berger AS, Kaplan AG. Clinical experience with the surgical removal of sub-foveal neovascular membranes. Ophthalmology 1992;99:969-76.

18 Bergink GJ, Deutman AF, van den Broek JFCN, van der Maazen RWM. Radiation therapy for subfoveal choroidal neovascular membranes in age-related macular degeneration. Graefes Arch Clin Exp Ophthalmol 1994;232:591-8.

19 Bergink GJ, Deutman AF, van den Broek JE, van Daal WA, van der Maazen RM. Radiation therapy for age-related van der Maazen RM. Radiation therapy for age-related subfoveal choroidal neovascular

20 Friedrichsen EJ, Slater JD, Loredo LN, Yonemoto LT, Inj J, Garcia CA, et al. Proton beam irradiation of subfovea choroidal neovascularisation in age-related macular degen eration. Invest Ophthalmol Vis Sci 1995;36(suppl):1020.

21 Immonen I, Jakkola A, Heikkonen J. Treatment of subfovea choroidal neovascular membranes using strontium 90 plaques irradiation. Invest Ophthalmol Vis Sci 1995; 36(suppl): 1022.

22 Finger PT. Radiotherapy for subretinal neovascularisation. Invest Ophthalmol Vis Sci 1995;36(suppl):1021.

23 Aristizibal S, Caldwell WK, Avilla J. The relationship of time-dose fractionation factors to complications in the treatment of pituitary tumors by irradiation. Int $\mathcal{F}$ Radia Biol Phys 1977;2:667-73.

24 Wara W, Irvine AR, Neger RE, Howes EL, Phillips TL Radiation retinopathy. Int $f$ Radiat Biol Phys 1979;5:81-3. 25 Miller ML, Goldberg SH, Bullock JD. Radiation retinopathy after standard radiotherapy for thyroid-related oph-

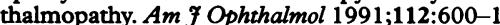

